\title{
Les parasitoses entériques. Problèmes de santé publique dans le département de la Réunion
}

\author{
par M. LARIVIERE, H. PICOT, G. LANGUILlat, B. BEAUVAIS, \\ M. THERIZOL et A. COUMBARAS
}

U.E.R. Lariboisière-St-Louis, 107, rue du Fbg-St-Denis, F 75010 Paris U.E.R. Hôtel-Dieu-Broussais, 15, rue de l'Ecole-de-Médecine, F 75006 Paris

\begin{abstract}
Résumé
Une enquête préliminaire effectuée en août 1969 dans le département de la Réunion montra un taux de parasitisme intestinal de $95 \%$ dans le groupe d'âge de 0 à 14 ans. Les trichocéphales sont les parasites dominant $(87 \%)$, puis les Ascaris (65\%), les Ankylostomes (23\%), les Lamblias (21 \%), Entamoeba coli (16\%), Endolimax nana (8\%), Anguillule (7\%), Entamoeba histolytica (5 \%). Les Oxyures, Taenia saginata et Taenia solium existent également mais n'ont pas été recherchés de façon systématique. L'importance des parasites intestinaux dans ce département d'Outre-Mer nous a conduit à déclencher un ensemble de mesures de lutte associant assainissement, éducation sanitaire et chimiothérapie. La création d'un laboratoire d'épidémiologie et d'hygiène du milieu doit permettre de mieux connaître les chaînes épidémiologiques et de surveiller les actions de prophylaxie.
\end{abstract}

\section{Summary}

A preliminary survey made in August 1969 in the department of La Réunion showed that the density of intestinal parasitism was $95 \%$ from birth to 14 years of age. Trichocephalus is the 
commonest parasite $(87 \%)$, then Ascaris (65\%), Ancylostoma (23\%), Lamblia (21\%), Entamoeba coli (16\%), Endolimax nana (8\%), Strongyloides (7\%), Entamoeba histolytica (5\%). Oxyurosis, Taenia saginata and T. solium are present too but have not been researched systematically.

The importance of intestinal parasitism in this overseas department led us to undertake series of measures combining clearance, heath education and chemotherapy.

The creation of a laboratory studying epidemiology of infection and problems of hygiene concerning local way of life, will give improved informations about exposure to infection and appreciation of prophylactic measures.

La fréquence du parasitisme intestinal chez l'homme est soulignée depuis de nombreuses années par les médecins de l'Ile de La Réunion et dans les rapports de la Direction de l'Action Sanitaire et Sociale. Mais la première évaluation statistique de cette endémie fut celle qu'effectua en 1957 une mission O.M.S. dont l'étude ne porta toutefois que sur la seule commune de Saint-André. Elle révéla l'existence d'un polyparasitisme chez presque tous les enfants d'âge scolaire et préconisa une lutte axée sur l'assainissement et l'éducation sanitaire de la population.

Depuis, des études plus fragmentaires ont confirmé l'existence d'une endémie importante dans d'autres parties de l'île.

Nous avons pu ainsi prendre connaissance de la thèse de Dominique Poissonnier (1959) faisant état d'observations personnelles à l'hôpital d'enfants de St-Denis, du travail des docteurs O.-M. Turquet au Centre Hospitalier départemental de Bellepierre et à l'hôpital d'enfants de Saint-Denis (1968), J. Farjon (1968) à l'hôpital de SaintLouis, Role (1968) dans le Service de Pédiatrie de Saint-Pierre et des communications orales de plusieurs autres confrères, lors d'un séjour que l'un de nous effectua à La Réunion en 1968 avec une équipe d'enseignants du Centre International de l'Enfance.

A notre retour à Paris, ayant eu l'occasion d'exposer, devant des responsables du Ministère de la Santé, les préoccupations de nos confrères réunionnais, face à une telle situation sanitaire, nous avons été chargés par le Ministère de la Santé Publique avec la participation de l'I.N.S.E.R.M. d'évaluer la situation réelle de l'endémie parasitaire entérique à La Réunion et d'étudier les méthodes d'action sanitaire à y opposer.

L'enquête préliminaire se déroula durant le mois d'août 1969.

Cette première mission dans l'île avait pour objet de rassembler les données et les moyens d'une enquête statistiquement valable pouvant être comparés à d'autres enquêtes à venir, nécessaires pour apprécier les effets de l'action sanitaire qui en découlerait.

Il nous a donc fallu faire l'inventaire des moyens matériels et en personnel pouvant être mis à notre disposition par le département (locaux, véhicules, personnel de laboratoire...) et, avec le service des statistiques (I.N.S.E.E.), établir l'échantillonnage de populations sur lequel porterait l'enquête. D'autre part, pour éprouver nos possi- 
bilités d'action sur le terrain et commencer la formation du personnel nécessaire, pour sélectionner les techniques coprologiques à utiliser, nous avons recueilli et examiné des échantillons de selles pris au hasard dans la presque totalité des communes de l'île.

Ce sont ces résultats que nous donnons ici, en précisant qu'ils n'ont donc pas de valeur statistique formelle, ni exhaustive, mais qu'en dépit de leur caractère aléatoire, ils sont comme nous le verrons très riches d'enseignements.

L'observation a porté sur 568 selles prélevées dans 17 communes sur les 24 que comporte l'île et sur des sujets de 4 à 15 ans.

\section{I. - Résultats}

\section{A) Infection globale :}

Les résultats font apparaître un taux d'infection de $95 \%$ qui est parmi les plus élevés dans le monde. Par ailleurs, $79 \%$ des sujets examinés sont polyparasités (17\% par plus de 3 parasites).

Ces résultats sont vraisemblablement même en dessous de la réalité. En effet, nous n'avons fait pour chaque selle qu'un examen direct et une méthode de concentration (technique de Ritchie). D'autres techniques ont été employées (Baermann, M.I.F. coloration, etc...), mais elles ne furent pas systématiques. De plus, nous n'avons pas utilisé la méthode de Graham (scotch-test), ce qui ne nous permet pas d'apprécier la fréquence du parasitisme par oxyures et taenias.

\section{B) Fréquence des différents parasites :}

On trouve à La Réunion la plupart des espèces parasitaires susceptibles d'habiter le tube digestif de l'homme, à l'exclusion des Trématodes que nous n'avons jamais rencontrés dans les échantillons examinés.

1. Dans le groupe des Nématodes à contamination orale, l'infection par Ascaris et Trichocéphale est de loin la plus élevée, puisque $93 \%$ des selles examinées, soit la quasi-totalité, contenaient l'un ou l'autre de ces parasites.

2. Parmi les Nématodes à contamination transcutanée, l'anguillule semble peu fréquente $(7 \%)$ en regard des ankylostomes $(23 \%)$. Mais rappelons que la technique de recherche des larves d'anguillules (extraction de Baermann) n'a été pratiquée que de manière occasionnelle au cours de cette enquête.

\begin{tabular}{lccc}
\hline \hline & $\begin{array}{c}\text { Ankylostome } \\
\text { ou } \\
\text { Anguillule }\end{array}$ & $\begin{array}{c}\text { Ascaris } \\
\text { ou } \\
\text { Trichocéphale }\end{array}$ & Protozoaires \\
\hline Sujets infectés $\ldots \ldots \ldots \ldots \ldots$ & $27 \%$ & $93 \%$ & $38 \%$ \\
\hline \hline
\end{tabular}

3. Les Protozoaires: $38 \%$ des selles examinées contenaient des protozoaires, dont $21 \%$ des lamblias et $5 \%$ des amibes. Il faut signaler là encore que la recherche 
d'Entamoeba histolytica a souffert des techniques frustes utilisées lors de l'enquête. L'amibiase est très certainement plus fréquente que ce qu'indiquent nos chiffres. Elle constitue d'ailleurs une des préoccupations majeures de nos confrères praticiens et nous en aurons une meilleure appréciation au terme de l'étude plus poussée actuellement en cours.

\section{C) Répartition géographique :}

L'étude de la distribution géographique des parasites selon les communes (cf. tableau) ne montre de différences marquantes que pour Ankylostomes et Anguillules.

Ceci tient aux particularités biologiques du cycle exogène de ces vers, très dépen. dants des facteurs hydroclimatiques. C'est ainsi que dans les communes « au vent», très humides, le pourcentage des cas d'infections par Ankylostomes et Anguillules est notablement plus élevé que dans la zone «sous le vent» aux précipitations moins abondantes.

\section{D) Facteurs favorisant l'infection parasitaire.}

Nous avions pensé, à l'aide d'un questionnaire rempli auprès des familles lors des récoltes de selles, pouvoir préciser le rôle joué par le promiscuité, les conditions d'hygiène de l'environnement et nous faire une idée de l'importance du parasitisme selon les classes d'âge des sujets examinés. Cette étude trop sommaire n'a finalement pas fourni de renseignements interprétables et nous la reprenons dans le cadre des zones d'observations que nous avons choisies en vue d'apprécier l'action sanitaire entreprise.

\section{E) Le problème du traitement antiparasitaire :}

Parmi les sujets dont nous examinions les selles, un grand nombre avait reçu un traitement anti-ascaridien (pipérazine et dérivés) dans les mois qui précédaient. Il nous a paru intéressant de tenir compte de ce facteur dans l'appréciation des résultats. Aussi avons-nous regroupé les sujets en 3 lots :

a) sujets ayant eu un traitement datant de moins de 2 mois ..... ( 87 sujets)

b) sujets ayant eu un traitement datant de plus de 2 mois ..... (169 sujets)

c) sujets n'ayant pas eu de traitement anti-ascaridien ........ (312 sujets) et nous avons comparé les pourcentages d'individus infectés par l'ascaris. Les résultats figurent dans le tableau suivant :

$\%$ de sujets infectés par Ascaris

\begin{tabular}{|c|c|}
\hline Traitement datant de moins de 2 mois $\ldots \ldots \ldots \ldots \ldots \ldots$ & 53 \\
\hline Traitement datant de plus de 2 mois $\ldots \ldots \ldots \ldots \ldots \ldots \ldots \ldots \ldots$ & 65 \\
\hline 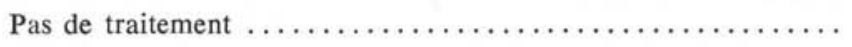 & 69 \\
\hline Echantillon total (568 selles) ............ & 65 \\
\hline
\end{tabular}


Le délai de deux mois a été choisi en fonction du cycle de l'ascaris : il faut qu'environ deux mois s'écoulent après l'infection pour que les œufs du parasite soient présents dans les selles. Ainsi, théoriquement, on ne doit pas retrouver d'œufs dans les selles d'un sujet traité dans les deux mois précédents.

Les résultats de cette comparaison appellent quelques commentaires :

- Il apparaît que le pourcentage de sujets infectés est plus faible pour ceux qui ont reçu un traitement récent que pour les autres.

- On peut constater d'autre part que la réinfection est quasi immédiate puisque après le délai de deux mois, le taux d'infection remonte à $65 \%$. Nous confirmons d'ailleurs ce phénomène par les résultats partiels que viennent de nous donner les zones d'observations.

Le fait important est que $53 \%$ des sujets traités depuis moins de deux mois restent parasités. Plusieurs explications sont possibles qui concourent à souligner l'inefficacité de l'action curative dans un contexte endémique d'une telle ampleur:

- le traitement de ces sujets ne comportait en général qu'une seule cure qui n'élimine pas tous les ascaris;

- ce traitement d'ailleurs ne résultait pas toujours d'une prescription médicale. La fréquence des infections helminthiques est connue de la plupart des familles réunionnaises qui assument souvent elles-mêmes leur propre déparasitage : soit en renouvelant périodiquement une ordonnance médicale sans consultation du praticien, soit par échange de « recettes » d'une famille à l'autre. Il en résulte des prescriptions fantaisistes sans grande efficacité ;

- mais l'échec du traitement est dominé par les réinfections permanentes, que ne peut contrarier aucune des thérapeutiques vermifuges ou vermicides actuellement connues. La pipérazine et ses dérivés n'agissent que sur les vers adultes du tube digestif et n'ont aucun effet sur la phase de migration larvaire qui suit directement l'infection.

Les tableaux suivants résument les données numériques établies par la présente enquête.

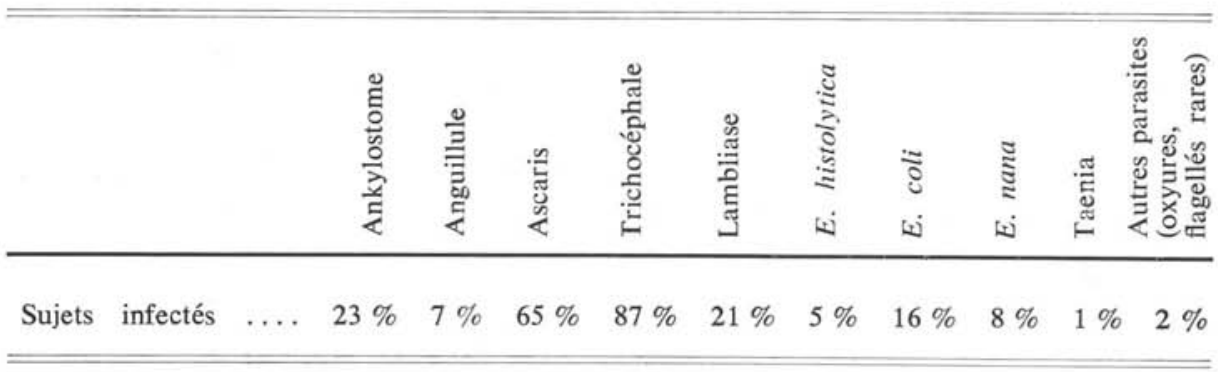

Résultats globaux selon les parasites

Annales de Parasitologie humaine et comparée (Paris), t. $48, \mathrm{n}^{\circ} 2$ 


\begin{tabular}{|c|c|c|c|c|c|c|c|c|c|c|c|}
\hline & 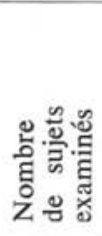 & $\frac{\text { ğ }}{\frac{0}{8}}$ & 兽 & 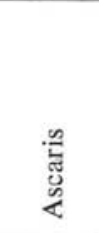 & 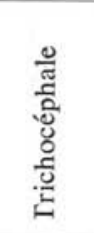 & 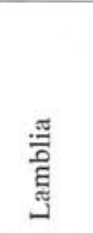 & 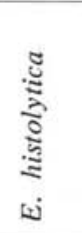 & $\begin{array}{l}\overline{0} \\
\text { ¿j }\end{array}$ & 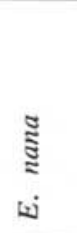 & 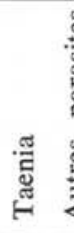 &  \\
\hline Zone sous le vent & 1921 & $13 \%$ & $5 \%$ & $59 \%$ & $83 \%$ & $27 \%$ & $5 \%$ & $15 \%$ & $7 \%$ & $0 \%$ & $3 \%$ \\
\hline Zone au vent .. & 376 & $28 \%$ & $20 \%$ & $68 \%$ & $91 \%$ & $19 \%$ & $5 \%$ & $17 \%$ & $8 \%$ & $8 \%$ & $1 \%$ \\
\hline
\end{tabular}

Résultats portant sur les deux principales zones climatiques

\section{II. - Commentaires}

De cette enquête partielle et des données recueillies dans les études antérieures, il est possible de dégager un certain nombre d'enseignements.

\section{A. La situation actuelle :}

Le chiffre de $95 \%$ de sujets parasités situe ce département d'outre-mer parmi les régions du monde au niveau d'hygiène le plus bas.

Toutefois, s'agissant d'un sondage et en raison des techniques coprologiques forcément limitées que nous avons utilisées et des moyens matériels assez précaires dont nous avons disposé, nous ne pouvons prétendre donner un inventaire exhaustif de toutes les parasitoses intestinales humaines présentes dans l'île.

Il est possible toutefois de dresser la première liste suivante :

1) Nématodes: Trichocéphales et Ascaris sont les parasites dominants avec les chiffres respectifs de $87 \%$ et $65 \%$ qui à eux seuls traduisent une importante pollution fécale du milieu.

L'infection par Oxyures est probablement importante. Nous l'avons mise en évidence dans plusieurs selles recueillies, mais sa fréquence ne peut être chiffrée qu'en utilisant une technique particulière (méthode de Graham) qui ne se justifiait pas dans ce type d'enquête.

Les Nématodes à transmission transcutanée sont également présents dans l'île de la Réunion et constituent une endémie élevée, malgré les taux apparemment bas d'Ankylostomes (23\%) et d'Anguillules (7\%) établis sur les résultats d'ensemble.

Leur distribution géographique n'est pas homogène, ce que laissaient prévoir au départ nos connaissances sur le cycle exogène particulier de ces parasites. S'ils sont absents de certaines zones sèches on les trouve au contraire avec une forte fréquence dans les régions humides. La répartition par commune révèle $60 \%$ de porteurs 


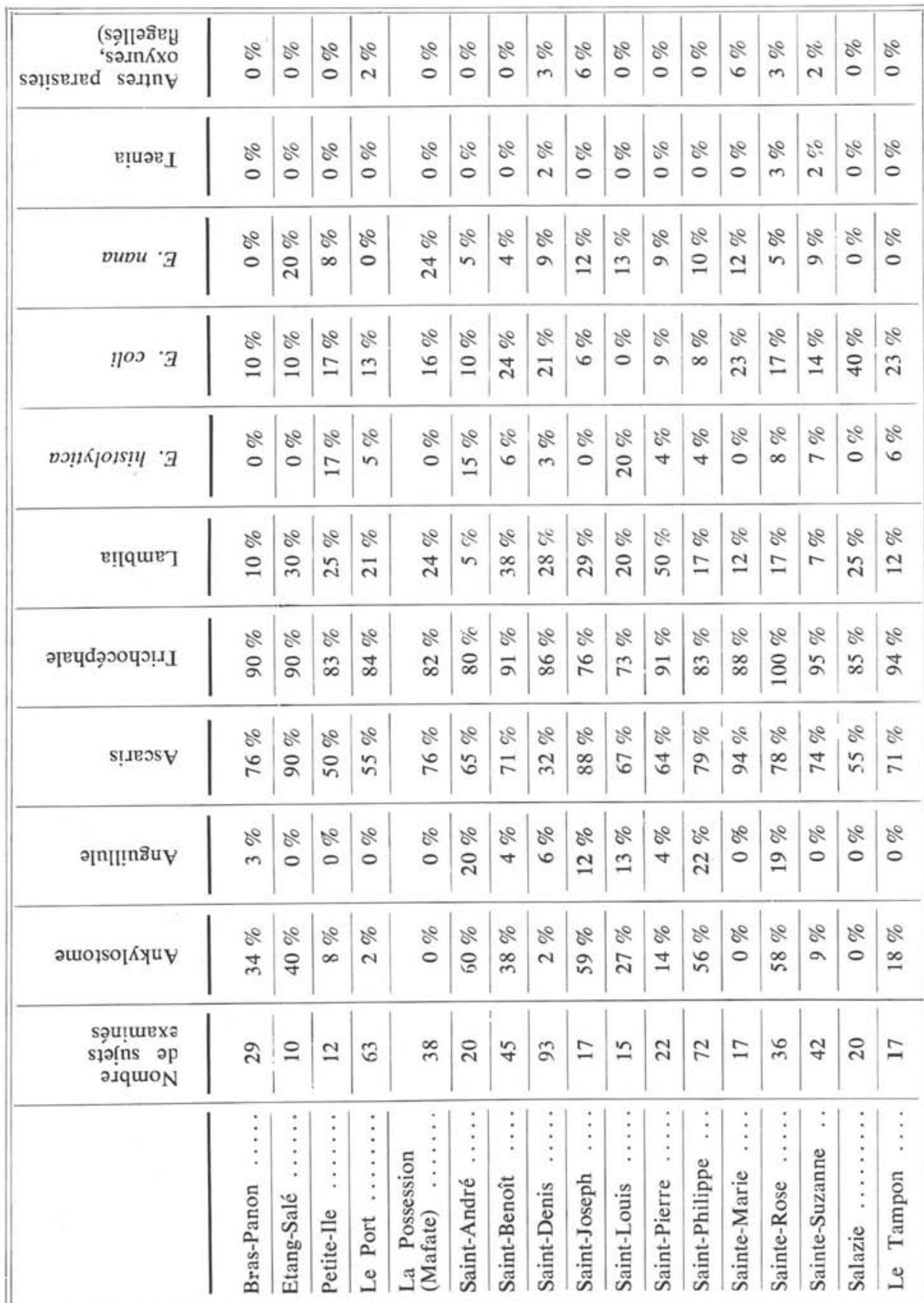


d'Ankylostomes à St-André, $56 \%$ à St-Philippe, $58 \%$ à Ste-Rose et à l'inverse $0 \%$ à la Possession et à Salazie, $2 \%$ au Port, $9 \%$ à Ste-Suzanne.

Il convient de signaler qu'après avoir procédé à quelques cultures de selles et à des examens de larves ou de vers adultes nous pouvons certifier que les deux espèces, Ankylostoma duodenale et Necator americanus sont présentes à La Réunion et semblent correspondre à des biotopes différents que nous préciserons dans des études ultérieures.

Pour l'anguillulose, les pourcentages d'infections obtenus sont largement inférieurs aux taux réels puisque la technique spécifique de Baermann a été rarement utilisée.

Néanmoins, nous constatons pour ce parasite les mêmes différences de fréquence entre les régions au vent et sous le vent: $0 \%$ à Etang Salé, Petite-Ile, Le Port, La Possession, contre $20 \%$ à St-André, $22 \%$ à St-Philippe, $19 \%$ à Ste-Rose.

2) Trématodes: Il n'existe aucun cas autochtone de bilharziose vésicale ou intestinale. Les rares observations enregistrées par nos confrères installés dans l'île concernent exclusivement des sujets venant de Madagascar où sévissent les deux bilharzioses ou de l'île Maurice, foyer de $S$. haematobium.

3) Cestodes: Nous n'avons pas recherché systématiquement les cas de taeniasis. A plusieurs reprises cependant, nous avons trouvé des œufs de taenia dans des échantillons de selles examinés sans qu'il soit possible sur leur seule morphologie d'affirmer leur appartenance à l'espèce Taenia saginata ou Taenia solium. Ce dernier existe sans aucun doute puisque les rapports de nos confrères neurologues ou psychiatres signalent la relative fréquence de cas de cysticercose constatés cliniquement, radiologiquement ou sérologiquement.

La présence et la fréquence de $T$. saginata restent donc à évaluer.

4) Protozonires : l'importance sur la morbidité et la mortalité générale des infections à protozoaires intestinaux est dominée par Entamoeba histolytica. L'identification précise de ce parasite est un examen délicat nécessitant des techniques d'enrichissement spécifiques que nous n'avons pas eu souvent les moyens d'utiliser ici. Il ne nous est donc pas possible maintenant de donner une image épidémiologique précise de l'endémie amibienne. Elle paraît grande comme en témoignent déjà les statistiques hospitalières surtout des services de pédiatrie qui signalent chaque année plusieurs cas d'amibiase dès l'âge du nourrisson.

Notre étude coprologique sur le terrain, tout incomplète qu'elle soit, révèle elle aussi des indices inquiétants d'infection amibienne dans certaines communes: $17 \%$ à Petite-Ile, $15 \%$ à Saint-André, $20 \%$ à Saint-Louis.

Nous avons reconnu également la présence de la plupart des autres protozoaires parasites du tube digestif. Les uns, Entamoeba coli, Endolimax, Pseudolimax, Embadomonas, Enteromonas, dénués de rôle pathogène, les autres, Trichomonas et surtout Lamblias, dont le pouvoir pathogène n'est plus discuté, confirment, s'il en était besoin, l'importance du péril fécal. 


\section{III. - Conséquences de cette importante endémie parasitaire entérique}

Elles sont extrêmement lourdes, tant pour l'individu que pour la collectivité.

$1^{\circ}$ L'individu est très souvent affecté par des manifestations aiguës, invalidantes ou même mortelles (anémies graves, complications chirurgicales telles que syndromes occlusifs, péritonites, abcès du foie...).

Il est toujours handicapé par les effets induits du parasitisme :

- accroissement du déficit protidique: des études O.M.S., rapport technique $1967, \mathrm{n}^{\circ} 379$, ont montré que chez des enfants hébergeant 13 à 40 ascaris et dont la ration alimentaire quotidienne comportait de 35 à 40 grammes de protéines, 4 grammes de protéines étaient perdus chaque jour par effet direct du parasite sur la digestion et l'absorption. Aussi, les bénéfices d'une amélioration du régime alimentaire sont-ils diminués ;

- accroissement du déficit vitaminique et de la malnutrition ;

- ralentissement du développement staturo-pondéral ;

- troubles du comportement (apathie profonde, nervosisme, méningisme vermineux) ;

- effets multiplicateurs des phénomènes morbides.

$2^{\circ}$ La charge imposée à la collectivité par une telle situation est écrasante. En effet, au coût direct que représentent les journées d'hospitalisation (plusieurs dizaines de milliers par an), la consommation médicamenteuse et la prise en charge des arrêts de travail prolongés, s'ajoutent des effets induits non quantifiables, retard scolaire de l'enfant, attitude générale de passivité apparente, faible productivité du Réunionnais, tous facteurs disqualifiant l'offre du travail.

Citons pour La Réunion les chiffres suivants :

- Au cours du $1^{\text {er }}$ semestre 1970 , il y eut, dans le service de pédiatrie (73 lits) de l'hôpital Félix-Guyon de Saint-Denis, 867 hospitalisations. 189 enfants (soit plus de 1 sur 5) ont été hospitalisés pour parasitose seule et 76 pour dénutrition associée à des parasitoses. Les 189 enfants ont entraîné 4725 journées d'hospitalisation dont le coût se chiffre à 33216750 francs C.F.A. Si l'on ajoute à ce chiffre les 76 parasitoses associées et dans certains cas déclenchantes de la dénutrition, on arrive pour le seul premier semestre 1970 à un coût de 45871750 francs C.F.A.

- Une étude menée auprès de la société d'importation des pharmaciens réunis a montré que le volume de médicaments antiparasitaires stricts ayant été mis en circulation durant l'exercice 1969-1970 se chiffrait à 100 millions de francs. 


\section{IV. - Les actions à entreprendre}

Elles nous ont été confiées par M. le Préfet et la Direction de l'Action Sanitaire et Sociale (D.A.S.S.).

Elles résultent de l'analyse des données épidémiologiques et de l'inventaire des moyens disponibles.

\section{LES DONNÉES ÉPIDÉMIOLOGIQUES :}

L'endémie parasitaire entérique est la conséquence de la dispersion anarchique sur le sol des matières fécales humaines. Selon la nature de ce sol, ses propriétés physico-chimiques, les données climatiques (ensoleillement, humidité...), les formes parasitaires (kystes, œufs, larves) contenues dans les selles trouveront les conditions favorables à leur développement et leur résistance. Elles sont à l'origine de contaminations nouvelles, de surinfections ou de réinfections après avoir souillé les mains, l'eau, les légumes (contamination orale) ou par contact direct de l'homme avec le sol ou l'eau (pénétration cutanée des larves infectantes d'Ankylostomes, d'Anguillules).

A ces facteurs de contamination directe s'ajoute la possibilité de transmission mécanique à distance des parasites présents sur le sol par l'intermédiaire des mouches, des blattes, des rongeurs, des animaux domestiques ou d'élevage.

Les conditions telluriques et bioclimatiques de La Réunion sont favorables au développement de la plupart des espèces parasitaires. Le sous-développement de l'équipement sanitaire en matière d'assainissement, l'ignorance par la population des données les plus élémentaires de l'hygiène expliquent le haut degré de l'endémie.

Inventaire des moyens disponibles: trois types d'actions peuvent être envisagés. Nous les analyserons, puis nous expliquerons les raisons de notre choix.

- Action médicamenteuse.

- Assainissement et hygiène du milieu.

- Education sanitaire.

\section{I. - L'action médicamenteuse.}

Elle consiste en l'administration de médicaments spécifiques destinés à détruire les parasites chez les sujets reconnus comme porteurs. Elle ne peut s'effectuer que sur prescription et surveillance médicales et doit s'appuyer sur des contrôles de laboratoire répétés et coûteux. C'est la méthode de lutte traditionnelle pratiquée dans ce département.

Ses limites:

1. On ne dispose pas pour l'instant d'un produit actif à la fois sur toutes les espèces parasitaires. La fréquence des cas de polyparasitisme constatée dans l'Ile contraint donc le médecin à faire appel à plusieurs types de médicaments prescrits successivement, ce qui allonge les délais du déparasitage. Ceci se comptabilise en outre 
par une multiplication des consultations médicales, des examens de laboratoire et des prescriptions médicamenteuses.

2. Aucun médicament antiparasitaire ne possède une efficacité à $100 \%$, ce qui réduit encore l'efficience du déparasitage.

3. Les anthelminthiques agissent sur les vers adultes, mais n'ont aucune action sur les formes infectantes, ni sur les formes de migrations larvaires intraorganiques qui précèdent l'installation des vers adultes dans le tube digestif. La thérapeutique n'a donc aucune valeur préventive, de sorte qu'un sujet soumis au traitement peut se trouver en même temps en cours de réinfection.

4. Dans le cadre présent de nos conceptions médicales, l'action curative ne se traduit en fait que par une succession d'actes isolés réalisés à la demande du malade qui éprouve les manifestations pathologiques du parasitisme. Il en résulte que les " porteurs sains » échappent au déparasitage et continuent à assurer la dissémination parasitaire.

A cette médecine individuelle on pourrait adjoindre des campagnes thérapeutiques de masse et en espérer théoriquement des résultats meilleurs. Mais ceux qui ont été obtenus dans les pays où elles ont été tentées, ne sont pas convaincants et se sont même à peu près partout soldés par des échecs. Les raisons en ont été : tolérés ;

- l'absence de médicaments multivalents, d'administration simple et parfaitement

— l'impossibilité de traiter systématiquement et en même temps tous les porteurs de parasites ;

- surtout la longue durée de conservation des parasites dans le milieu extérieur. Ainsi l'œuf d'ascaris assure son complet développement sur le sol en deux semaines à une température de $30^{\circ} \mathrm{C}$ ou 4 semaines à une température de $20^{\circ} \mathrm{C}$ et peut ensuite conserver son pouvoir infectant durant un an. De même, la larve infectante d'ankylostome peut résister à 6 à 18 mois dans la nature.

Pour toutes ces raisons, l'action thérapeutique fatalement limitée dans le temps ne peut contrecarrer à elle seule la réinfection à partir d'un milieu qui peut demeurer si longtemps contaminé.

\section{En résumé :}

- Le traitement médicamenteux conserve toute sa justification dans la lutte contre les manifestations pathologiques du parasitisme.

- Il est essentiellement curatif et ne peut même, sous la forme de campagne de masse, parvenir à rompre définitivement la chaîne épidémiologique de transmission.

- Le traitement systématique d'une collectivité peut toutefois, dans certains cas, être envisagé en complément d'opérations ponctuelles d'assainissement. 


\section{II. - Assainissement et Hygiène du milieu.}

Ils se fixent pour objectifs :

- l'arrêt de la pollution fécale du milieu ;

- la mise à la disposition de la population de moyens et de principes d'hygiène préventifs lui permettant d'échapper à la contamination. Ces deux actions sont étroitement liées et doivent être menées conjointement.

Cette forte prévalence des parasitoses entériques à La Réunion est la conséquence de deux phénomènes d'ailleurs dépendants l'un de l'autre:

1) Permanence de l'ensemencement parasitaire du milieu. La majorité des habitants de La Réunion élimine ses matières fécales n'importe où sur le sol (cours, jardins, champs, ravines...) ou dans des installations précaires (feuillées, tinettes...) qui n'en assurent pas la neutralisation. Ils pérennisent de la sorte la contamination du milieu.

2) Facilité et fréquence de la contamination des individus. Les modes de vie familiale et sociale favorisent la dispersion des formes infestantes des parasites. Tous les circuits de la chaîne alimentaire peuvent être contaminés. L'eau à usage domestique est souvent non potable. La culture maraîchère n'est pas contrôlée et l'usage de l'engrais animal ou humain reste encore très répandu. Porcs et volailles sont rarement stabulés et sont susceptibles d'assurer la dissémination mécanique à distance des parasites. Il en est de même des chiens et des chats. Enfin, l'environnement immédiat de l'habitat se caractérise par un éparpillement anarchique des ordures ménagères favorable à la multiplication des mouches, des blattes et des rongeurs.

La disparition de l'endémie parasitaire ne peut donc être espérée que dans des actions drastiques sur le milieu comportant:

- la mise à la disposition de tous de l'eau potable ;

- le contrôle de la qualité hygiénique des légumes offerts à la consommation ,

- l'hygiène de l'habitat ;

- l'élimination des matières fécales par le tout à l'égout, les fosses septiques ou en attendant la mise en place de ces coûteuses installations, la construction pour chaque famille de latrines correctes, de prix de revient faible.

Durant l'année 1972, plus de 4000 latrines à fond perdu ont été mises en service dans différentes parties de l'île à la demande des intéressés. Plus de 6000 sont prévues cette année. Leur originalité réside dans le fait que la plaque recouvrant la fosse et traditionnellement en béton est fabriquée par une usine locale à partir de fibres de canne à sucre, de bagasse, conglomérée et plastifiée. Grâce aux subventions annuelles obtenues des caisses d'Allocations familiales et de Sécurité sociale, ces plaques sont données gratuitement à toute famille en faisant la demande mais ayant en charge le creusement et l'aménagement de la fosse. 


\section{III. - Education sanitaire de la population.}

C'est un préalable en même temps qu'une nécessaire mesure d'accompagnement des actions d'assainissement.

La population doit devenir consciente de l'important préjudice porté à sa santé, par l'existence d'une si lourde endémie parasitaire. Elle doit être avertie des différentes voies de contamination et convaincue du bénéfice que lui apportera la construction et l'utilisation des latrines à bon marché qui lui sont proposées. Il lui faut sortir de l'attitude passive de l'inévitable d'autant qu'en ce domaine tout le succès dépend de sa compréhension et de sa discipline.

Les méthodes d'éducation sanitaire sont multiples et variées, mais le choix que l'on en fait demande à être éprouvé par un contact direct et permanent avec la population.

La création de l'Association réunionnaise d'éducation sanitaire et sociale (A.R.E.S.S.) a été décidée dans ce but. Présidée par le Docteur Michel Turquet, elle a multipié depuis un an ses actions en profondeur et trouvé une audience de plus en plus grande dans la population. C'est par elle que s'effectue la distribution de plaques de latrines et elle mène des études pour solutionner le problème de l'approvisionnement des familles en eau potable.

IV. - Le déroulement de si complexes opérations d'assainissement et d'hygiène du milieu imposait la mise en place d'un service d'études et de surveillance.

Nous avons donc obtenu la création d'un laboratoire d'épidémiologie et d'hygiène du milieu placé sous la direction du laboratoire de parasitologie de l'U.E.R. médicale de l'Université Paris VII. Il fonctionne depuis plus d'un an grâce à la présence permanente sur le terrain du Docteur $\mathrm{H}$. Picot qui en assume la direction pratique.

Nous nous sommes donnés pour objectif :

1) La réalisation d'une enquête destinée à établir sur des données statistiquement valables et reproductibles dans l'avenir :

- la prévalence des infections parasitaires, par zones climato-telluriques, par tranches d'âges, selon le type de l'habitation, etc...

Cette enquête est l'élément de connaissance de base qui permettrait de contrôler dans l'avenir les résultats de la campagne d'assainissement.

L'étude coprologique sera complétée par une enquête sur l'habitat, le mode de vie, en vue d'orienter les recherches expérimentales tendant à préciser les facteurs déterminants de la contamination.

Nous prévoyons également un bilan systématique du poids, de la taille, du taux de protides, de l'hémoglobine, du nombre des globules rouges pour évaluer l'incidence réelle des parasitoses sur la morbidité générale.

2) L'observation approfondie de zones témoins où seront testées périodiquement les méthodes d'assainissement et d'éducation sanitaire. 
3) Des recherches en laboratoire portant sur les cycles biologiques des parasites, leur sensibilité à différents agents biologiques ou chimiques, le contrôle de la pollution parasitaire des légumes, de l'eau, de l'engrais biologique, les techniques de désinfection.

\section{Conclusions}

L'endémie parasitaire entérique qui sévit à La Réunion est un fléau social grave tant pour l'individu que pour la collectivité.

Il exige que lui soit opposé un programme cohérent de mesures sanitaires spécifiques,

Il n'existe pas de solutions à court terme, ni l'espérance de solutions dans ce délai.

L'assainissement et l'hygiène du milieu appuyés sur l'éducation sanitaire de la population sont les seules actions logiques et rentables dans la perspective d'une éradication à long terme.

Les réalisations pratiques proposées sont exécutées dans le cadre de la Direction de l'Action Sanitaire et Sociale avec l'appui technique du laboratoire départemental d'Epidémiologie et d'Hygiène du Milieu et de l'Association Réunionnaise d'Action Sanitaire et Sociale. 